\title{
Upon the Utility of Spherical Harmonic Expansions in the Evaluation of Cluster Integrals ${ }^{1}$
}

\author{
George D. J. Phillies ${ }^{2}$
}

Received October 26, 1981

\begin{abstract}
A novel procedure for the analytic evaluation of cluster integrals is given. By means of a result of Silverstone and Moats which transforms the spherical harmonic expansion of a function around a given point into a new spherical harmonic expansion around a displaced point, a $3 \mathrm{~N}$-dimensional cluster integral for $N$ point particles $(N>2)$ may be reduced to $2 N+1$ trivial integrals and $N-1$ interesting integrals, an improvement over the usual reduction to six trivial integrals and $3 N-6$ nontrivial integrals. For hard spheres, the $N-1$ integrals involve only a series of simple polynomials taken between linear algebraic bounds.
\end{abstract}

KEY WORDS: Cluster integrals; spherical harmonics; virial coefficients; hard spheres.

\section{INTRODUCTION}

The pressure $P$ of an imperfect unionized gas may in general be expanded as a virtual series

$$
\frac{P V}{K_{B} T}=n\left(1+B_{2} \rho+B_{3} \rho^{2}+B_{4} \rho^{3}+\cdots\right)
$$

$n$ being the number of particles in the system, $\rho$ being the number density, and the $B_{j}$ being the virial coefficients. A major problem of equilibrium statistical mechanics is the calculation of the virial coefficients from the intermolecular potentials. As is well known, ${ }^{(1)}$ each $B_{N}$ may be obtained

\footnotetext{
${ }^{1}$ This work was supported in part by the National Science Foundation under Grant No. CHE79-20389.

${ }^{2}$ Department of Chemistry, The University of Michigan, Ann Arbor, Michigan 48109.
} 
from the potential energy in terms of the coordinates of $N$ particles. For short-range, two-body central forces, the expression for $B_{N}$ is a single $3 N$-dimensional integral. A major limitation of this theory is the difficulty in performing the multidimensional integrals. If one ignores surface effects, translational and rotational symmetry make the first six integrations (for $N=2$, the first five integrations) trivial; the remaining $3 N-6$ integrals are more challenging.

The form of the virial coefficients has been most extensively studied for the hard-sphere fluid, ${ }^{(2-5)}$ in which the sphere-sphere potential $U(r)$ is

$$
\begin{aligned}
& U(r)=+\infty, \quad e^{-\beta U(r)}=0 \quad(r \leqslant d) \\
& U(r)=0, \quad e^{-\beta U(r)}=1 \quad(r>d)
\end{aligned}
$$

$d$ being the sphere diameter and $\beta=\left(K_{B} T\right)^{-1}$. Values for $B_{2}$ through $B_{7}$ have been obtained by analytic and numerical means. For example,

$$
B_{3}=-\frac{1}{3 V} \int d \mathbf{r}_{1} d \mathbf{r}_{2} d \mathbf{r}_{3} f_{12} f_{13} f_{23}
$$

where

$$
f_{i j}=\exp \left[-\beta U\left(r_{i j}\right)\right]-1
$$

$\mathbf{r}_{i j}=\mathbf{r}_{i}-\mathbf{r}_{j}$, and $\mathbf{r}_{i}$ is the location of molecule $i$. This may be reduced to

$$
B_{3} \propto \int d r_{12} d r_{13} d r_{23} f_{12} f_{13} f_{23}
$$

where $r_{12}=\left|\mathbf{r}_{12}\right|$, where $r_{12}, r_{13}$, and $r_{23}$ must satisfy such triangle inequalities as $\left|r_{13}-r_{23}\right| \leqslant r_{12}<r_{13}+r_{23} . B_{4}$ may similarly be reduced to a sixfold integral over the distances between pairs from a set of four particles, where again the $r_{i j}$ must satisfy triangle inequalities. For $j>5$ the geometric constraints from the $f_{i j}$ are so complex that numerical and Monte-Carlotype procedures have generally been used to calculate the $B_{j}$.

In this paper, a new technique for the analytic evaluation of cluster integrals is demonstrated. For a cluster of $N$ point particles, one need only perform an $(N-1)$-fold integral. For clusters of simple topology, the integral is closed; for more complex clusters, infinite series (whose convergence is treated) can arise. The results given here are therefore similar to those of Katsura, ${ }^{(4)}$ but the integrals found here are more elementary, and the number of indices over which explicit summations are needed is smaller. The following section presents the general results, and applies them to a hard-sphere system.

In Section 3, the results are discussed and compared with those in the literature. 


\section{GENERAL RESULTS: HARD-SPHERE POTENTIAL}

For a system in which only additive two-body forces are important, an $N$-particle cluster integral may be written as a product of Mayer $f$ functions $f_{i j}$ integrated over all possible configurations of the $N$ particles in the cluster. The $f_{i j}$ are related to the intermolecular potential $U_{i j}$ via Eq. (1.4), each $f_{i j}$ being spherically symmetric about either $\mathbf{r}_{i}$ or $\mathbf{r}_{j}$. Our general line of attack is to expand each $f_{i j}(i, j \neq 1)$ in terms of coordinates $\mathbf{r}_{1 i}, \mathbf{r}_{1 j}$ measured from a single particle 1 , located at $\mathbf{r}_{1}$, and then integrate over the $\mathbf{r}_{1 l}$.

The expansion of a function $f\left(\mathbf{r}_{i j}\right)=f\left(\left|\mathbf{r}_{1 i}-\mathbf{r}_{1 j}\right|\right)$ in terms of $r_{1 i}$ and $r_{1 j}$ is a solved problem, at least in spherical polar coordinates. Silverstone and Moats $^{(6)}$ treat the general question of expressing a function of the form $F(\mathbf{r})=f(r) Y_{L M}(\theta, \phi)$ in terms of spherical harmonics and radial functions centered on a displaced origin, simplifying the previous results of Sharma. ${ }^{(7)}$ It is shown by Silverstone and Moats that a displaced function for $F(\mathbf{r})$ may be expanded as

$$
\begin{aligned}
& F(\mathbf{r}-\mathbf{R})=\sum_{\substack{l=0 \\
l+L+\lambda \text { even }}}^{\infty} \sum_{\lambda=|l-L|}^{l+L} \nu_{l \lambda L}(r, R) \\
& \times \sum_{m=-l}^{l} C_{\lambda L M l m} Y_{\lambda, M-m}\left(\theta_{R}, \phi_{R}\right) Y_{l m}(\theta, \phi) \\
& C_{\lambda L M l m}=\int d \Omega_{r} Y_{\lambda, M-m}^{*}\left(\theta_{r}, \phi_{r}\right) Y_{l m}^{*}\left(\theta_{r}, \phi_{r}\right) Y_{L M}\left(\theta_{r}, \phi_{r}\right) \\
& \nu_{l \lambda L}(r, R)=\frac{2 \pi(-1)^{l}}{R} \sum_{a=0}^{(L+l+\lambda) / 2} \sum_{b=0}^{(L+l+\lambda-2 a) / 2} D_{l \lambda L a b}\left(\frac{r}{R}\right)^{2 b-l-1} \\
& \times \int_{|r-R|}^{r+R}\left(\frac{r^{\prime}}{R}\right)^{2 a-L+1} f\left(r^{\prime}\right) d r^{\prime} \\
& D_{l \lambda L a b}=1 /[(2 a) ! !(2 a-2 L-1) ! !(2 b-2 l-1) ! !(L+l+\lambda-2 a-2 b) ! ! \\
& \times(2 b) ! !(L+l-\lambda-2 a-2 b-1) ! !] \\
& (2 N) ! !=2^{N} N ! \\
& (2 N-1) ! !=(2 N) ! /(2 N) ! ! \\
& (-2 N-1) ! !=(-1)^{N} /(2 N-1) ! !
\end{aligned}
$$

Here $\Omega_{r}$ is the angular part of the spherical polar coordinate $\mathbf{r}$. The $Y_{l m}(\theta, \phi)$ follow the normalization of Edmonds, ${ }^{(8)}$ so in particular $Y_{l m}^{*}(\theta, \phi)$ $=(-1)^{m} Y_{l,-m}(\theta, \phi)$. 
By means of Eq. (2.1), and use of relative coordinates $r_{1 j}$ any $N$ particle cluster integral $\int d \mathbf{r}_{1} d \mathbf{r}_{2}, \ldots, d \mathbf{r}_{N}[\ldots$ product of $f$ functions $\ldots]$ may be put into the form $\int d \mathbf{r}_{1} d \mathbf{r}_{12}, \ldots, d \mathbf{r}_{1 N}[G]$, where in [G] (1) all $f$ functions involving particle 1 are left undisturbed, and (2) all $F_{i j}$ with $i \neq 1$ and $j \neq 1$ are rewritten in terms of the $\nu_{l \lambda L}\left(\left|\mathbf{r}_{1 i}\right|,\left|\mathbf{r}_{1 j}\right|\right)$, multiplied by spherical harmonics centered on $\mathbf{r}_{1}$. The resulting integrals $\int d \Omega_{1 r}$ over products $Y_{l m}\left(\Omega_{1 r}\right) Y_{l^{\prime} m^{\prime}}\left(\Omega_{1 r}\right) \ldots$ are, however, trivial, and exist in tabulated form. As will be seen below, in many cases one finds $l, m=l^{\prime}, m^{\prime}=\cdots \equiv 0,0$, eliminating sums over the indices $l$ and $m$. On performing the angular integrals, one is left with $\int d \mathbf{r}_{1}$, which is the volume of the system, and $N-1$ integrals over the distances $d r_{12} \ldots d r_{1 N}$. By these steps, the original $3 N$-dimensional integral can be reduced to an $(N-1)$-dimensional form.

The hard-sphere potential [Eq. (1.2)] is now used to generate examples for spheres of unit diameter: $f_{i j}=0\left(r_{i j}>1\right)$ and $f_{i j}=-1\left(r_{i j} \leqslant 1\right)$. The spherical harmonic expansion of $f_{i j}$, for coordinates centered on $\mathbf{r}_{1}$, is

$$
\begin{aligned}
& f_{i j}=-(4 \pi)^{1 / 2} Y_{00}(\Omega), \quad r_{i j} \leqslant 1 \\
& f_{i j}=0, \quad r_{i j}>1
\end{aligned}
$$

Since $f_{i j}$ is a sphere, in Eqs. (2.1) one has $L=M=0$, and $l=\lambda$. Equations (2.1) then become

$$
\begin{gathered}
F(\mathbf{r}-\mathbf{R})=\sum_{l=0}^{\infty} \nu_{l l 0}(r, R) \sum_{m=-l}^{l} C_{l 00 l m} Y_{l,-m}\left(\Omega_{R}\right) Y_{l m}\left(\Omega_{r}\right) \\
\nu_{l l 0}^{(R, r)}=\frac{2 \pi(-1)^{l}}{R} \sum_{a=0}^{l} \sum_{b=0}^{l-a} D_{l l 0 a b}\left(\frac{r}{R}\right)^{2 b-l-1} \int_{|r-R|}^{r+R}\left(\frac{r^{\prime}}{R}\right)^{2 a+1} f\left(r^{\prime}\right) d r^{\prime}
\end{gathered}
$$

For small $l, v_{l l 0}$ has simple forms:

$$
\begin{gathered}
\nu_{000}^{(r, s)}=\frac{\pi}{r s}\left(\min \left\{\begin{array}{c}
\left.(r+s)^{2}\right\} \\
1
\end{array}\right\}-\min \left\{\begin{array}{c}
(r-s)^{2} \\
1
\end{array}\right)\right)\left[-(4 \pi)^{1 / 2}\right] \\
\nu_{l l 0}(r, s)=0, \quad l>0, \quad r+s<1 \quad \text { or } \quad|r-s|>1 \\
\nu_{110}(r, s)=\frac{\pi}{4 r^{2} s^{2}}\left[1-2 r^{2}-2 s^{2}-2 r^{2} s^{2}+r^{4}+s^{4}\right]\left[-(4 \pi)^{1 / 2}\right] \\
r+s \geqslant 1, \quad|r-s| \leqslant 1 \\
v_{220}(r, s)=\frac{\pi}{8 r^{3} s^{3}}\left[\left(1-r^{2}-s^{2}\right)^{3}-4 r^{2} s^{2}\left(1-r^{2}-s^{2}\right)\right]\left[-(4 \pi)^{1 / 2}\right] \\
r+s \geqslant 1, \quad|r-s| \leqslant 1
\end{gathered}
$$

where $\min \{\}$ takes the value of the lesser of its two arguments.

We begin with the two-point function $B_{2}=(2 V)^{-1} \int d \mathbf{r}_{2} d \mathbf{r}_{3} f_{23}$. For the sake of demonstration, $f_{23}$ is expanded about an arbitrary third point $\mathbf{r}_{1}$, 
with $\mathbf{r}=\mathbf{r}_{2}-\mathbf{r}_{1}, \mathbf{s}=\mathbf{r}_{3}-\mathbf{r}_{1}$, so

$$
B_{2}=-\frac{1}{2 V} \int d \mathbf{r} d \mathbf{s} \sum_{l=0}^{\infty} v_{l l 0}(r, s) \sum_{m=-l}^{l} C_{l 00 l m} Y_{l m}^{*}\left(\Omega_{s}\right) Y_{l m}\left(\Omega_{r}\right)
$$

The angular integrals vanish unless $l, m=0,0 . \int d \Omega Y_{00}=(4 \pi)^{1 / 2}$, while $C_{00000}=(4 \pi)^{-1 / 2}$. For a spherical system with a hard outer wall, the radial integrals have limits 0 and $L, L$ being the radius of the complete system. Since $B_{2}$ is symmetric in $r$ and $s$, we only need treat the region with $r>s$. $\nu_{000}(r, s)$ vanishes for $r-s>1$, so

$$
\begin{aligned}
& B_{2}=V^{-1} 4 \pi^{2} \int_{1}^{L} d r \int_{r-1}^{r} d s r s\left\{\min \left\{(r+s)^{2}\right\}-(r-s)^{2}\right\} \\
& +V^{-1} 4 \pi^{2} \int_{0}^{1} d r \int_{0}^{r} d s r s\left\{\min \left\{(r+s)^{2}\right\}-(r-s)^{2}\right\}
\end{aligned}
$$

The limits on the integral are so arranged that $(r-s)^{2} \leqslant 1$. Noting $V$ $=4 \pi L^{3} / 3$, Eq. (2.6) is

$$
B_{2}=2 \pi / 3+O\left(L^{2} / V\right)+O\left(V^{-1}\right)
$$

The term in $L^{2} / V$ is a surface correction, while $2 \pi / 3$ is the conventiona ${ }^{(9)}$ value for $B_{2}$. Of greater interest is the third virial coefficient

$$
B_{3}=-\frac{1}{3 V} \int d \mathbf{r}_{1} d \mathbf{r}_{2} d \mathbf{r}_{3} f_{12} f_{13} f_{23}
$$

which can be expanded as

$$
B_{3}=\frac{-1}{3} \int d \mathbf{r} \int d \mathbf{s} f(r) f(s) \sum_{l=0}^{\infty} \nu_{l l 0}(r, s) \sum_{m=-l}^{l} C_{l 00 l m} Y_{l m}^{*}\left(\Omega_{s}\right) Y_{l m}\left(\Omega_{r}\right)
$$

The angular integrals again vanish for $l>0$, so Eq. (2.9) is

$$
B_{3}=\frac{4 \pi^{2}}{3} \int_{0}^{1} d r \int_{0}^{1} d s r s\left(\min \left\{\begin{array}{c}
(r+s)^{2} \\
1
\end{array}\right\}-(r-s)^{2}\right)=\frac{5}{8} \cdot\left(\frac{2 \pi}{3}\right)^{2}
$$

which is the well-known value for the third virial coefficient of a system of hard spheres. However, the reduction from Eq. (2.8) to Eq. (2.10) has left one with a two-dimensional integral, rather than the three-dimensional integral one might have expected. The simplification becomes more spectacular if one considers a cluster of four particles.

Using the customary symbolic notation, the fourth virial coefficient can be expanded in terms of closed cluster integrals

$$
\begin{gathered}
\boldsymbol{B}_{4}=-8 V^{-1} \int d \mathbf{r}_{1} d \mathbf{r}_{2} d \mathbf{r}_{3} d \mathbf{r}_{4}[ \\
{\left[f_{12} f_{23} f_{34} f_{41}+6 f_{12} f_{23} f_{34} f_{41} f_{13}\right.} \\
\left.+f_{12} f_{13} f_{14} f_{23} f_{24} f_{34}\right]
\end{gathered}
$$


Some perturbation theory calculations also make use of open cluster integrals such as $\int d \mathbf{r}_{2} d \mathbf{r}_{3} f_{12} f_{23} f_{34}$ or $\int d \mathbf{r}_{2} d \mathbf{r}_{3} f_{12} f_{23} f_{34} f_{13} f_{24}$. The first diagram in $B_{4}$, hereinafter ${ }^{(10)}$ denoted $D_{4}$, is

$$
D 4=V \int d \mathbf{r} d \mathbf{s} d \mathbf{t} f(\mathbf{r}) f(\mathbf{r}-\mathbf{s}) f(\mathbf{s}-\mathbf{t}) f(\mathbf{t})
$$

where $\mathbf{t}=\mathbf{r}_{1}-\mathbf{r}_{4}$. On separate expansion of $f(\mathbf{r}-\mathbf{s})$ and $f(\mathbf{r}-\mathbf{t})$

$$
\begin{aligned}
D 4= & V \int d \mathbf{r} d \mathbf{s} d \mathbf{t} f(\mathbf{r}) f(\mathbf{t}) \sum_{l, l=0}^{\infty} \sum_{m=-l}^{l} \sum_{m^{\prime}=-l^{\prime}}^{l_{l l}}(r, s) \nu_{l^{\prime} l^{\prime} 0}(s, t) \\
& \times C_{l 00 l m} C_{l^{\prime} 00 l^{\prime} m^{\prime}} Y_{l m}^{*}\left(\Omega_{r}\right) Y_{l m}\left(\Omega_{s}\right) Y_{l^{\prime} m^{\prime}}^{*}\left(\Omega_{s}\right) Y_{l^{\prime} m^{\prime}}\left(\Omega_{t}\right)
\end{aligned}
$$

The integral over $\Omega_{s}$ vanishes for $l m \neq l^{\prime} m^{\prime}$ because of the orthogonality of the $Y_{l m}$. The angular parts of the integrals on $r$ and $t$ vanish for $l m \neq 0$ and $l^{\prime} m^{\prime} \neq 0 . D 4$ is then

$$
\begin{aligned}
D 4= & -4 \pi^{3} V \int_{0}^{1} d r \int_{0}^{2} d s \int_{0}^{1} d t r t\left[\min \left\{\begin{array}{c}
(r+s)^{2} \\
1
\end{array}\right\}-\min \left\{(r-s)^{2}\right\}\right] \\
1 & \times\left[\min \left\{\begin{array}{c}
(s+t)^{2} \\
1
\end{array}\right\}-\min \left\{\begin{array}{c}
(s-t)^{2} \\
1
\end{array}\right\}\right]
\end{aligned}
$$

One notes that $D_{4}$ is an integral over simple polynomials between simple bounds. The presence of the $\min \{\}$ function will complicate the calculation, but no further thought about the details of solid geometry is required. One has

$$
D 4=-4 \pi V \int_{0}^{1} d r\left[\pi^{2}\left(-\frac{5}{6}+\frac{r^{2}}{2}-\frac{r^{4}}{10}+\frac{r^{6}}{630}\right)\right]=\left(\frac{2 \pi}{3}\right)^{3}(2.59047619)
$$

in agreement with the known result. ${ }^{(9)}$ The term of Eq. (2.15) within the square brackets is the open cluster integral $(d 3)_{12}$ (for $r_{12} \leqslant 1$ ) of Rowlinson. ${ }^{(3)}$

The fully connected four-point integral

$$
D 6=\int d \mathbf{r}_{1} d \mathbf{r}_{2} d \mathbf{r}_{3} d \mathbf{r}_{4} f_{12} f_{13} f_{21} f_{23} f_{24} f_{34}
$$

presents a new level of complication. Substitution of Eq. (2.1) shows

$$
\begin{aligned}
D 6= & V \int d \mathbf{r} d \mathbf{s} d \mathbf{t} f(r) f(s) f(t) \sum_{l, l^{\prime}, l^{\prime \prime}=0}^{\infty} \sum_{m, m^{\prime}, m^{\prime \prime}} v_{l 0}(r, s) \nu_{l^{\prime} l^{\prime}}(t, s) \\
& \times \nu_{l^{\prime \prime} l^{\prime \prime} 0}(t, r) Y_{l m}^{*}\left(\Omega_{r}\right) Y_{l m}\left(\Omega_{s}\right) Y_{l^{\prime} m^{\prime}}^{*}\left(\Omega_{t}\right) Y_{l^{\prime} m^{\prime}}\left(\Omega_{s}\right) Y_{l^{\prime \prime} m^{\prime \prime}}^{*}\left(\Omega_{t}\right) Y_{l^{\prime \prime} m^{\prime \prime}}\left(\Omega_{r}\right)
\end{aligned}
$$


For each angular integral the integrand is of the form $Y_{l m}^{*}(\Omega) Y_{l^{\prime} m^{\prime}}(\Omega)$; these vanish for $1 m \neq l^{\prime} m^{\prime}$. Since each angular argument appears twice, the sixfold sum on the $l$ and $m$ collapses as far as a single sum on $l$, but no further. For $D 6$, one obtains a triple integral over an infinite series

$$
D 6=-V \int_{0}^{1} d r \int_{0}^{1} d s \int_{0}^{1} d t r^{2} s^{2} t^{2} \sum_{l=0}^{\infty} \nu_{l l 0}(r, s) \nu_{l l 0}(s, t) \nu_{l l 0}(r, t)(2 l+1)
$$

the limits on $r, s$, and $t$ deriving from the $f_{1 j}$ functions. The integral is substantially simplified by using the symmetry between $r, s$, and $t$ to integrate only over the region with $1 \geqslant r \geqslant s \geqslant t \geqslant 0$. The first terms of 2.18 are $D 6(l=0)=(2 \pi / 3)^{3}(1.025669627) V$ and $D 6(l=1)=(2 \pi / 3)^{3}$ $(0.102656250) V$; the exact value is $D 6$ (total) $=V \cdot(2 \pi / 3)^{3}(1.2669040)$. The first two terms amount to $89 \%$ of the total.

The rapidity of convergence of the power series in $l$ should not be overstated. Since $v_{l l 0}(r, s)(l>0)$ vanishes unless $r+s>1$, the range over which $\nu_{110}$ is integrated is far less than that over which $\nu_{000}$ is integrated. By using the symmetry of $D 6$ between $r, s$, and $t,(2.18)$ reduces to

$$
\begin{aligned}
D 6= & -6 V \int_{0}^{1} d r \int_{0}^{r} d s \int_{0}^{s} d t[l=0 \text { term }] \\
& -6 V \int_{1 / 2}^{1} d r \int_{1 / 2}^{r} d s \int_{1-s}^{s} d t[l>0 \text { terms }]
\end{aligned}
$$

If one considers only the more restricted range of integration, one finds 0.62645 and 0.10266 for the contribution of the $l=0$ and the $l=1$ terms, respectively.

If one did not perform the outermost integral (on $r$ ), but did integrate $s$ and $t$ over their complete ranges, one would gain an expression for the two-point open cluster integral corresponding to $D 6$. Because of the $\min \{\}$ functions in the $\nu_{l l 0}(r, s)$, the two-point function must be tabulated separately for $r<1 / 2$ and $r \in(1 / 2,1)$. Such a tabulation would be pointless unless enough terms in $\nu_{l l o}(r, s)$ had been included to give a reasonable numerical value for $D 6$ itself.

With clusters of more complicated topology, a single summation on $l$ is not enough. The fully connected five-point cluster $E 10$ (in Rowlinson's notation) is first worked as an example; some general rules relating clusters to their angular integrals are then set down. One has

$$
E 10=\frac{1}{-30 V} \int d \mathbf{r}_{1} d \mathbf{r}_{2} d \mathbf{r}_{3} d \mathbf{r}_{4} d \mathbf{r}_{5} f_{12} f_{13} f_{14} f_{15} f_{23} f_{24} f_{25} f_{34} f_{35} f_{45}
$$

Using the condensed notation $\nu_{(5)}(r, s) \equiv \nu_{l s, l 5,0}(r, s), C_{5} \equiv C_{l_{5} 00 l_{5} m_{5}}$, and 
$Y_{5}\left(\Omega_{r}\right) \equiv Y_{l_{5} m_{s}}\left(\Omega_{r}\right)$, and the new internal coordinate $u=r_{5}-r_{1}$, one has

$$
\begin{aligned}
E 10= & (1 / 30) \int d \mathbf{r} d \mathbf{s} d \mathbf{t} d \mathbf{u} f(r) f(s) f(t) f(u) \sum_{l_{1} m_{1}, l_{2} m_{2}, l_{3} m_{3}, l_{4} m_{4}, l_{5} m_{5}, l_{6} m_{6}} \\
& \times \nu_{(1)}(r, s) Y_{1}^{*}\left(\Omega_{r}\right) Y_{1}\left(\Omega_{s}\right) \nu_{(2)}(r, t) Y_{2}^{*}\left(\Omega_{r}\right) Y_{2}\left(\Omega_{t}\right) \nu_{(3)}(r, u) Y_{3}^{*}\left(\Omega_{r}\right) Y_{3}\left(\Omega_{u}\right) \\
& \times \nu_{(4)}(s, t) Y_{4}^{*}\left(\Omega_{s}\right) Y_{4}\left(\Omega_{t}\right) \nu_{(5)}(s, u) Y_{5}^{*}\left(\Omega_{s}\right) Y_{5}\left(\Omega_{u}\right) \nu_{(6)}(t, u) Y_{6}^{*}\left(\Omega_{t}\right) Y_{6}\left(\Omega_{u}\right) \\
& \times \prod_{j=1}^{6}(-1)^{m_{j}} C_{j}
\end{aligned}
$$

The angular integrals are all over products of three $Y_{l m}(\Omega)$ functions, and therefore introduce the Wigner $3-j$ symbols

$$
\begin{aligned}
\int d \Omega Y_{l_{1} m_{1}}(\Omega) Y_{l_{2} m_{2}}(\Omega) Y_{l_{3} m_{3}}(\Omega)= & {\left[\frac{\left(2 l_{1}+1\right)\left(2 l_{2}+1\right)\left(2 l_{3}+1\right)}{4 \pi}\right]^{1 / 2} } \\
& \times\left(\begin{array}{ccc}
l_{1} & l_{2} & l_{3} \\
0 & 0 & 0
\end{array}\right)\left(\begin{array}{lll}
l_{1} & l_{2} & l_{3} \\
m_{1} & m_{2} & m_{3}
\end{array}\right)
\end{aligned}
$$

Tables of $3-j$ and higher symbols and formulas for their numerical calculation are readily available. Substituting $f(r)$ for a hard sphere, and noting that each pair $l_{j} m_{j}$ appears in precisely two $3-j$ symbols,

$$
\begin{aligned}
E 10= & -\frac{1}{480 \pi^{2}} \int_{0}^{1} d r \int_{0}^{1} d s \int_{0}^{1} d t \int_{0}^{1} d u r^{2} s^{2} t^{2} u^{2} \sum_{\substack{l m_{1}, l m_{2}, l m_{3}, l m_{4}, l m_{5}, l m_{6}}} \nu_{(1)}(r, s) \nu_{(2)}(r, t) \nu_{(3)} \\
& \times(r, u) \nu_{(4)}(s, t) \nu_{(5)}(s, u) \nu_{(6)}(t, u)\left(\begin{array}{ccc}
l_{1} & l_{2} & l_{3} \\
0 & 0 & 0
\end{array}\right)\left(\begin{array}{ccc}
l_{1} & l_{2} & l_{3} \\
-m_{1} & -m_{2} & -m_{3}
\end{array}\right) \\
& \times\left(\begin{array}{ccc}
l_{1} & l_{4} & l_{5} \\
0 & 0 & 0
\end{array}\right)\left(\begin{array}{ccc}
l_{1} & l_{4} & l_{5} \\
m_{1} & -m_{4} & -m_{5}
\end{array}\right)\left(\begin{array}{ccc}
l_{2} & l_{4} & l_{6} \\
0 & 0 & 0
\end{array}\right)\left(\begin{array}{ccc}
l_{2} & l_{4} & l_{6} \\
m_{2} & m_{4} & -m_{6}
\end{array}\right) \\
& \times\left(\begin{array}{ccc}
l_{3} & l_{5} & l_{6} \\
0 & 0 & 0
\end{array}\right)\left(\begin{array}{lll}
l_{3} & l_{5} & l_{6} \\
m_{3} & m_{5} & m_{6}
\end{array}\right) \prod_{j=1}^{6}\left[C_{j}\left(2_{j}+1\right)\right]
\end{aligned}
$$

A further substantial simplification is possible by rewriting the products of $3-j$ symbols in terms of the $6-j$ symbol, for which tabulated values also exist. From Edmonds, the $6-j$ symbol is

$$
\begin{aligned}
\left\{\begin{array}{lll}
j_{1} & j_{2} & j_{3} \\
j_{4} & j_{5} & j_{6}
\end{array}\right\}= & \sum_{\text {all } m}\left(\begin{array}{lll}
j_{1} & j_{2} & j_{3} \\
m_{1} & m_{2} & m_{3}
\end{array}\right)\left(\begin{array}{ccc}
j_{1} & j_{5} & j_{6} \\
-m_{1} & m_{5} & m_{6}
\end{array}\right) \\
& \times\left(\begin{array}{ccc}
j_{4} & j_{2} & j_{6} \\
m_{4} & -m_{2} & -m_{6}
\end{array}\right)\left(\begin{array}{ccc}
j_{4} & j_{5} & j_{3} \\
-m_{4} & -m_{5} & -m_{3}
\end{array}\right) \\
& \times(-1)^{\sum_{i=1}^{6}\left(j_{i}+m_{i}\right)}
\end{aligned}
$$


Identifying $j_{1}, j_{2}, j_{3}, j_{4}, j_{5}$, and $j_{6}$ with $l_{1}, l_{3}, l_{2}, l_{6}, l_{4}$, and $l_{5}$, respectively, noting that the $3-j$ symbols change phase during an odd permutation of columns by a factor $(-1)^{j_{1}+j_{2}+j_{3}}$, and rearranging the $3-j$ symbols with $m=0$ to cancel the phase changes arising from the rearrangement of (2.23) into a product of the form of (2.24), the eight $3-j$ symbols of Eq. (2.23) have the reduced expression

$$
\left(\begin{array}{ccc}
l_{1} & l_{3} & l_{2} \\
0 & 0 & 0
\end{array}\right)\left(\begin{array}{ccc}
l_{1} & l_{4} & l_{5} \\
0 & 0 & 0
\end{array}\right)\left(\begin{array}{ccc}
l_{6} & l_{4} & l_{2} \\
0 & 0 & 0
\end{array}\right)\left(\begin{array}{ccc}
l_{6} & l_{3} & l_{5} \\
0 & 0 & 0
\end{array}\right)\left\{\begin{array}{lll}
l_{1} & l_{3} & l_{2} \\
l_{6} & l_{4} & l_{5}
\end{array}\right\}
$$

the $6-j$ symbol eliminating the sum on $m$, the remainder of Eq. (2.23) being unaffected. Each of the $3-j$ symbols in expression (2.25) will vanish if the sum of its indices is odd; if the sum is even, each symbol can immediately be expressed as a product of factorials [Ref. 9, Eq. (3.7.17)].

While the polynomial series implied by Eq. (2.23) are somewhat bulky for paper-and-pencil manipulation, the integral involves only products of polynomials taken between numerical or linear polynomial bounds. Equations (2.23) and (2.18) are therefore in a form particularly well-suited for evaluation by means of the symbolic integration routines now becoming available on digital computers.

A general method for doing all of the angular parts of an $N$-hardsphere cluster integral is now apparent: Sketch the symbolic diagram for the cluster integral; choose the most highly connected point in the cluster as the origin particle 1 . For each line connecting a pair of particles $i, j(i, j$ both $>1$ ) one gains a sum on the indices $l_{i j} m_{i j}$ and a factor $v_{(i j)}\left(\left|r_{1 i}\right|,\left|r_{1 j}\right|\right)$, the subscripts $(i j)$ being $l_{i j} l_{i j} 0$. The angular integral on $\Omega_{j}$ is found by counting the numbers of lines to particle $j$, not including any connection to particle 1 . If only one such line exists, the indices $l_{j i}, m_{j i}$ for that particle $j$ have the unique value 0 . If two such lines exist, their corresponding indices $l, m$ are set equal to each other, but are not further constrained. If three such lines exist, one introduces for their indices a set of $3-j$ symbols as seen in Eq. (2.22). One notes from Eq. (2.3a) that $m_{i j}$ appears explicitly both as $+m_{i j}$ and as $-m_{i j}$, a contingency dealt with by systematically entering into the $3-j$ symbol the term $+m_{i j}$ if $i>j$ and $-m_{i j}$ if $i<j$. The partition function, of which each cluster integral is a part, does not change if one rotates the coordinate system; the explicit summations over the $m_{i j}$ should therefore not be necessary. These sums may be eliminated by use of $6-j$ symbols and the orthogonality relations for the $3-j$ symbols.

For diagrams of sufficient complexity (the fully connected six-particle cluster $F 15$ is perhaps the simplest example) one must treat angular averages over products of four spherical harmonics, as treated implicity in Ref. 8, which argues that all of the more complicated angular averages over spherical harmonics can be expressed entirely in terms of $6-j$ symbols. These averages will not be treated at this time. 


\section{DISCUSSION}

It is of course true that simplifying the calculations of cluster integrals is not the same as trivializing that calculation. To compute an $N$-particle cluster integral, one still needs to evaluate an $(N-1)$-dimensional integral. For the hard-sphere system, this can be done analytically, if tediously. If one wants to proceed to analytic calculations using, e.g., the LennardJones potential or other $r^{-n}$ potential, one has to cope algebraically with the strong singularity in $\exp \left[-a r^{-n}\right]$ near $r=0$. For modeling reality, one may be better off with a clean analytic form for $f(r)$ rather than $V(r)$. Quantum mechanical many-electron calculations of a realistic $V(r)$ are only likely to give this function in numerical form, so that using realistic values of $V(r)$ to determine $f(r)$ of $\nu_{l l^{\prime} 0}(r)$ will only be possible numerically. In a calculation $B_{N}$ for a series of values of $N$, the integrals in the definition of $v_{l o}(R, r)$ need only be performed once. After determining $G(m, a)$ $=\int_{0}^{a}\left(r^{\prime}\right)^{m} f\left(r^{\prime}\right) d r^{\prime}$ in steps of the integration mesh size for various $m, G(m, a)$ may be used to compute all the $v_{l l 0}$. We have not investigated this point further.

For most potentials, the results presented here will reduce the dimensionality of the integrals defining the $B_{N}$; the final $N-1$ integrations will still need to be done numerically. It may well be easier to do numerical integration of an $(N-1)$ - rather than a $(3 N-6)$-dimensional integral, regardless of whether optimized Monte Carlo or true numerical integration procedures are used, at least if the computer can use symbolic manipulation schemes to determine the exact forms of the integrals.

The results of Section 2 for the fully connected four-particle cluster are in many ways similar to those of Katsura, who obtained a value for $D 6$ for the hard sphere + attractive square well potential by means of Fourier transforms, using intermediate expressions for $D 6$ in terms of products of spherical Bessel functions and Gegenbauer polynomials. On performing the angular integrations, Katsura's method gives a three-dimensional integral over a triply infinite (3-index) power series. Summation of the nine lowest terms in the series gave good agreement $(0.1 \%)$ with the known hard-sphere value for D6. Many of Katsura's intermediate formulas, such as the integrals over products of spherical Bessel functions, are similar to the intermediate formulas which appear in the work of Silverstone ${ }^{(10)}$ and Silverstone and Moats, ${ }^{(6)}$ on which this work is based. The substantial differences between the results given here for hard spheres and the results of Katsura are as follows: (i) The intermediate calculations here require a lower level of analytic sophistication and (ii) the generality of application of the technique has perhaps been made more apparent.

The use of spherical harmonic expansions in equilibrium statistical mechanics is not altogether new. One notes, for example, the work of Steele 
and coworkers, ${ }^{(11-14)}$ Blum and Torruella, ${ }^{(15)}$ Sandler, ${ }^{(16)}$ and Jepsen and Friedman. ${ }^{(17)}$ Much of this work deals primarily with the existence of orientation and angle-dependent interactions, and their effect on translational and orientational pair correlation functions and on the second virial coefficient. Chen and Steele ${ }^{(14)}$ do treat third and fourth virial coefficients, and the corresponding contributions to the pair distribution function, in the Percus-Yevick approximation, using a Fourier transform technique similar to that of Katsura. The results in Refs. 11-16 thus largely do not make use of multicenter integrals of the sort whose simplification has here been discussed. The work of Jepsen and Friedman treats angle-dependent interactions, using a Fourier transform technique to examine ring and ringlike diagrams as they relate to solute-solvent-solute forces. Since the fundamental equation (2.3) can be used to expand functions $f(r) Y_{L M}(\Omega)$ for $L M \neq 00$, a direct extension of the results of this paper may in principle be applied in the calculation of higher coefficients of nonspherical particles.

\section{REFERENCES}

1. J. E. Mayer, Statistical Mechanics (Wiley, New York, 1977).

2. J. J. van Laar, Proc. R. Acad. Sci. (Amsterdam) 1:273 (1899).

3. J. S. Rowlinson, Proc. R. Acad. Sci. (London) A279:147 (1964).

4. S. Katsura, Phys. Rev. 115: 1417 (1959).

5. F. H. Ree and W. G. Hoover, J. Chem. Phys. 46:4181 (1967).

6. Harris J. Silverstone and Richard K. Moats, Phys. Rev. A 16:1731 (1977).

7. R. R. Sharma, Phys. Rev. A 13:517 (1976).

8. A. R. Edmonds, Angular Momentum in Quantum Mechanics (Princeton University Press, New Jersey, 1974).

9. D. A. McQuarrie, Statistical Mechanics (Harper and Row, New York, 1976).

10. Harris J. Silverstone, J. Chem. Phys. 47:537 (1967).

11. W. A. Steele, J. Chem. Phys. 39:3197 (1963).

12. R. Pecora and W. A. Steele, J. Chem. Phys. 42:1872 (1965).

13. J. R. Sweet and W. A. Steele, J. Chem. Phys. 47:3022 (1967); J. Chem. Phys. 47:3029 (1967).

14. Y-D. Chen and W. A. Steele, J. Chem. Phys. 50:1428 (1969).

15. L. Blum and A. J. Torruella, J. Chem. Phys. 56:303 (1972).

16. S. I. Sandler, Molecular Phys. 28:1207 (1974).

17. D. W. Jepsen and H. L. Friedman, J. Chem. Phys. 38:846 (1963). 